\title{
A PULSED POLARIZED ELECTRON SOURCE FOR ATOMIC PHYSICS EXPERIMENTS
}

\author{
C.D. SCHRÖTER, A. DORN, R. MOSHAMMER, C. HÖHR AND J. ULLRICH \\ Max-Planck-Institut für Kernphysik, Saupfercheckweg 1, 69117 Heidelberg, \\ Germany
}

\begin{abstract}
Recently, a pulsed photoelectron source has been set up to satisfy the beam specifications required for atomic collision studies using so-called "reaction microscopes". The gun is in operation and delivers short electron pulses $(\sim 500 \mathrm{ps})$ using GaAs/AlGaAs heterostructure crystals. Illuminating the photocathode with light from a pulsed laser diode an average electron current of $60 \mu \mathrm{A}$ has been obtained. A quantum efficiency of $3 \%$ and a cathode lifetime of one week have been achieved.

Planned future atomic collision measurements require a cold beam of transversely polarized electrons. First design changes have been made to produce short pulses of monochromatic and spin-polarized electrons in the future.
\end{abstract}

\section{Introduction}

Kinematically complete experiments for multiple ionization of atoms and molecules by electron impact give detailed information about the role of the electron-electron interaction. In our group great efforts are undertaken to study these collisions systematically at incident electron energies $\left(\mathrm{E}_{0} \sim 100 \mathrm{eV}\right)$ near ionization threshold. Furthermore first ionization experiments in the presence of a strong laser field $\left(\sim 10^{13} \mathrm{~W} / \mathrm{cm}^{2}\right)^{1,2,3}$ as well as quantum mechanically complete experiments, like ionization and/or excitation in collisions of spin-polarized electrons with polarized targets, are in preparation.

All these electron-impact ionization experiments are performed using the cold-target recoil-ion momentum spectroscopy $y^{4,5,6,7}$. This successful experimental method demands, however, short-pulsed electron beams of high quality. Optimum conditions require pulse widths of less than $500 \mathrm{ps}$ (for "laserassisted" experiments even as short as $\sim 100 \mathrm{ps}$ ), a beam diameter of $100 \mu \mathrm{m}$ at the target and a pulse-repetition rate of up to $3 \mathrm{MHz}$.

In the recent past, a pulsed photoelectron source has been developed and set up ${ }^{8,9}$ satisfying the beam specifications required by our "reaction microscope" experiments ${ }^{6,7}$. 


\section{Experimental Set-up}

The experimental set-up of the electron source and details of the preparation technique of the photocathodes are described elsewhere ${ }^{8,9}$. Therefore only a short description will be given here. A schematic diagram of the set-up is shown in figure 1 . The source consists of a three-chamber UHV system, i.e. a

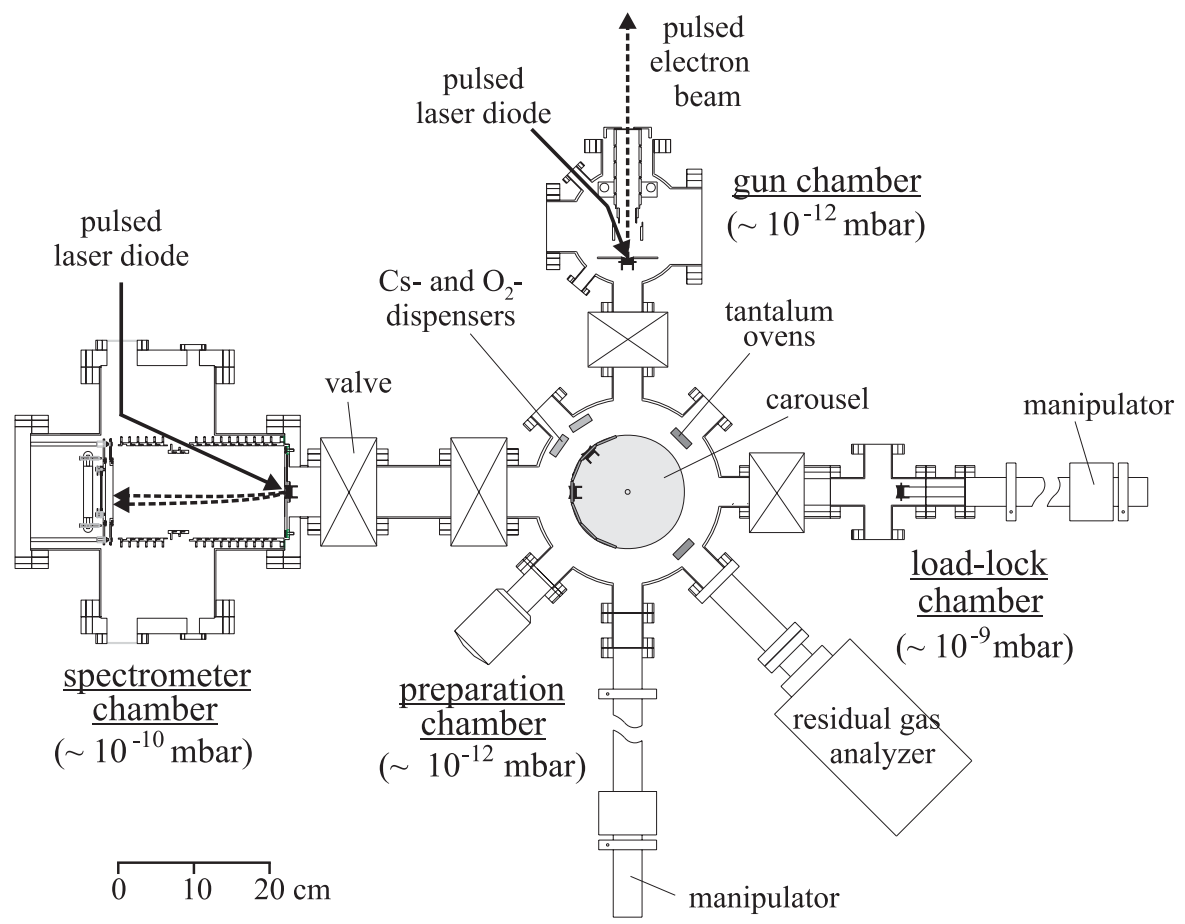

Figure 1. Experimental set-up of the pulsed electron source.

load-lock chamber, a photocathode-preparation chamber and a gun chamber. Both, the preparation and gun chamber are operating at a base pressure in the $10^{-12}$ mbar regime, the load-lock chamber at $\sim 10^{-9}$ mbar. This threechamber UHV system allows the fast exchange of photocathodes within a few minutes without breaking the ultra high vacuum of the gun chamber.

The photocathodes are prepared by an oxide-free technique ${ }^{10}$ and are transported without exposure to air to the preparation chamber. After a heatcleaning procedure the GaAs crystals are activated with cesium and oxygen 
to obtain a negative electron affinity. The activated photocathodes are finally transferred to the gun chamber.

In the gun chamber the cathode is illuminated in reflection mode with a pulsed laser diode (up to $3.3 \mathrm{~mW}$ ) operating at a wavelength of $650 \mathrm{~nm}$. An average emission current of $60 \mu \mathrm{A}$, i.e. a quantum efficiency of $3 \%$, and a photocathode lifetime of one week have been achieved. Short pulses of electrons are produced using GaAs/AlGaAs heterostructure crystals with a thin active GaAs layer. At the layer thickness of $0.9 \mu \mathrm{m}$ the long tail of the electron pulse, generated by a $\delta$-pulse light excitation, extends out to less than $200 \mathrm{ps}^{11}$. Adding the laser diode pulse width of 100 to $300 \mathrm{ps}$, which depends on the laser power, a total electron-pulse width of less than $500 \mathrm{ps}$ is expected.

\section{Perspectives}

Atomic collision experiments using cold electrons as projectiles, as well as transversely polarized electrons, are planned in the future. Design changes of the electron gun are already in progress to produce a monochromatic and even shorter pulsed $(\sim 100 \mathrm{ps})$ electron beam. The gun chamber will be replaced by a new UHV chamber, which recently has been designed. A $90^{\circ}$-electrostatic deflector for the production of transversely polarized electrons has been built and will be mounted inside the new chamber together with an electron optics for the beam transport. The new construction should allow in a further step even cooling of the photocathodes.

The production of an intense cold electron beam with a high degree of polarization demands a powerful infrared laser system. We have built a tuneable Ti:Sapphire laser $(670-1070 \mathrm{~nm})$ that fits these requirements, delivering $600 \mathrm{~mW}$ at a pulse width of 100 to $200 \mathrm{fs}$. Using strained GaAs photocathodes together with circularly polarized light from this laser system the source should provide in the future a beam of spin-polarized electrons with a high degree of polarization (up to $80 \%$ ).

\section{Acknowledgments}

This work was partially supported by the Deutsche Forschungsgemeinschaft within the Leibniz-program and the SFB 276. The heterostructure material was kindly put at our disposal by A.S. Terekhov. We are grateful to A.S. Terekhov as well as to A. Wolf and D. Orlov for their continuous support.

PST2001: submitted to World Scientific on November 7, 2001 


\section{References}

1. D. Khalil, A. Maquet, and R. Taïeb, C. J. Joachain and A. Makhoute, Phys. Rev. A 56, 4918 (1997).

2. F. Ehlotzky, A. Jaroń, J.Z. Kamiński, Phys. Rep. 297, 63 (1998).

3. C. J. Joachain, M. Dörr, N. Klystra, Adv. At. Mol. Opt. Phys. 42, 225 (2000).

4. R. Moshammer, M. Unverzagt, W. Schmitt, J. Ullrich, H. SchmidtBöcking, Nucl. Instr. and Meth. B 108, 425 (1996).

5. J. Ullrich, R. Moshammer, R. Dörner, O. Jagutzki, V. Mergel, H. Schmidt-Böcking and L. Spielberger, J. Phys. B 30, 2917 (1997).

6. A. Dorn, R. Moshammer, C.D. Schröter, T. J.M. Zouros, W. Schmitt, H. Kollmus, R. Mann, and J. Ullrich, Phys. Rev. Lett. 82, 2496 (1999).

7. A. Dorn, A. Kheifets, C. D. Schröter, B. Najjari, C. Höhr, R. Moshammer, and J. Ullrich, Phys. Rev. Lett. 86, 3755 (2001).

8. C. D. Schröter, A. Dorn, R. Moshammer and J. Ullrich, International Workshop on Polarized Sources and Targets, Erlangen 1999, Conference Proceedings, 292 (1999).

9. C. D. Schröter, A. Dorn, J. Deipenwisch, C. Höhr, R. Moshammer and J. Ullrich, International Workshop on Polarized Electron Source and Polarimeters, Nagoya 2000, in SPIN 2000, AIP Conference Proceedings 570, 996 (2001).

10. A.S. Terekhov, private communication.

11. P. Hartmann, J. Bermuth, D. v. Harrach, J. Hoffmann, S. Köbis, E. Reichert, K. Aulenbacher, J. Schuler, and M. Steigerwald, J. Appl. Phys. 86, 2245 (1999). 